\title{
Study on frequency and predictors of dementia after ischemic stroke: the Chongqing Stroke Study
}

David Zhou*

\begin{abstract}
Address: 2nd Department of Neurology, Daping Hospital, 3rd Military Medical University, China
\end{abstract}
* Corresponding author

from International Society on Brain and Behaviour: 2nd International Congress on Brain and Behaviour Thessaloniki, Greece. 17-20 November 2005

Published: 28 February 2006

Annals of General Psychiatry 2006, 5(SuppI I):SI 95 doi:I0.1 I86/I744-859X-5-SI-SI 95

\section{Background}

We studied a large hospitalized cohort of patients aged 55 years and over with acute ischemic stroke to identify the frequency and predictors of poststroke dementia.

\section{Materials and methods}

A total of 434 consecutive patients with ischemic stroke were enrolled in this study. During admission, the demographic data, vascular risk factors, stroke features, and neurological status information were collected. All subjects were examined by a battery of neuropsychological tests during admission and 3 months after stroke. Logistic regression analysis was used to find the predictors of poststroke dementia.

\section{Results}

(1) The frequency of poststroke dementia was $27.2 \%$, that of stroke-related dementia was $21.6 \%$, and that of dementia after first-ever stroke was $22.7 \% 3$ months after stroke. (2) Univariate analysis indicated that older age, low educational level ( $<$ or $=6$ years), everyday drinking, diabetes mellitus, atrial fibrillation, prior stroke, left carotid territory infarction, embolism, multiple stroke lesions, dysphasia, and gait impairment were more frequent in the patients with poststroke dementia. (3) Multivariate analyses demonstrated that age (OR 1.179, 95\%CI 1.1301.230), low educational level (OR 1.806, 95 \%CI 1.0243.186 ), everyday drinking (OR 3.447, $95 \% \mathrm{CI} 1.591-$ 7.468), prior stroke (OR 2.531, $95 \% \mathrm{CI}$ 1.419-4.512), atrial fibrillation (OR 3.475, 95\%CI 1.712-7.057), dysphasia (OR 5.873, $95 \%$ CI 2.620-13.163), and left carotid territory infarction (OR 1.975, 95\%CI 1.1523.388) were associated with poststroke dementia.

\section{Discussion}

The frequency of dementia is about one-forth of patients with ischemic stroke 3 months after stroke. Independent predictors of poststroke dementia include age, low educational level, everyday drinking, prior stroke, dysphasia, atrial fibrillation, and left carotid territory infarction.

\section{References}

I. Pohjasvaara T: Clinical determinants of poststroke dementia. Stroke 1998, 29:75-8I. 